\title{
Angiotensin-Converting Enzyme 2, Feedback Systems, and COVID-19
}

\author{
Miklos P. Salgo, PhD, MD
}

ABSTRACT: Angiotensin-converting enzyme (ACE) and angiotensin-converting enzyme 2 (ACE2) are key components of the renin-angiotensin system (RAS), which maintains homeostasis of blood pressure, among other functions. ACE acts in a positive feedback loop, providing amplification, while ACE2, acting in a negative feedback loop, modulates the ACE action, providing stability and fine control. Certain demographic groups that are more likely to become infected with SARS-CoV-2, the virus that causes COVID-19-and once infected, to have more severe disease and higher mortality-have a deficiency of ACE2. ACE2 has recently been shown to be a major receptor by which SARS-CoV-2 enters cells. This entry process is associated with downregulation of ACE2. Thus, viral cell entry per se can interfere with the RAS feedback system. Viral penetration of cells using ACE2 can lead to a shift in the RAS from the usual "preventive" ACE2 axis to the "adverse" ACE axis of the feedback systems. This imbalance, with predominance of the adverse ACE axis, can lead to downstream actions of increased vasoconstriction, inflammation, and thrombosis. These, in turn, can affect end organs, initiating COVID-19-associated conditions such as cytokine storm, thrombosis, multisystem inflammatory syndrome in children, and acute respiratory distress syndrome. This focused review highlights the importance of feedback systems in our understanding of the disruption of ACE2 feedback and resulting clinical consequences in COVID-19.

KEYWORDS: Angiotensin-converting enzyme 2 (ACE2), renin-angiotensin system (RAS), coronavirus, COVID-19, cybernetics, feedback systems

Feedback is a central feature of life processes.... The process of feedback governs how we grow, respond to stress and challenge, and regulate factors such as body temperature, blood pressure, and cholesterol level. This apparent purposefulness ... operates at every level - from the interaction of proteins in cells to the interactions of organisms in complex ecologies.

From Exploring the Way Life Works: The Science of Biology ${ }^{1}$

\section{AFFILIATIONS:}

Infectious diseases and internal medicine (retired), Hillsborough, California CITATION:

Salgo MP. Angiotensin-converting enzyme 2, feedback systems, and COVID-19. Consultant. Published online October 6, 2020. doi:10.25270/ con.2020.10.00006

Received July 17, 2020. Accepted August 15, 2020.

\section{DISCLOSURES:}

The author reports no relevant financial relationships.

\section{CORRESPONDENCE:}

Miklos Salgo, PhD, MD (miklossalgo@aol.com)
Simple causal reasoning about a feedback system is difficult because the first system influences the second and the second system influences the first, leading to a circular argument. This makes reasoning based on cause and effect tricky, and it is necessary to analyze the system as a whole.

From Feedback Systems: An Introduction for Scientists and Engineers $^{2}$

A ngiotensin-converting enzyme (ACE) and angiotensin-converting enzyme 2 (ACE2) are well characterized in the renin-angiotensin system (RAS) for maintaining blood pressure $(\mathrm{BP})$ homeostasis. In normal conditions, $\mathrm{ACE}$, via a positive feedback loop, provides amplification, while ACE2, via a negative feedback loop, modulates the ACE loop and provides stability and fine control. ACE2 has recently gained attention in SARS-CoV-2 infection as the main mechanism of viral entry into cells. This viral entry is associated with downregulation of ACE2, disrupting its feedback loop. ${ }^{3}$ ACE2 is also a key factor in other systems, often with complex interactions. 


\section{Table 1. The Renin-Angiotensin System (RAS)}

\section{ACE Adverse Axis}

Ang $\mathrm{II}$ is the major driver of the adverse axis, increasing ACE and BP.

- Renin cleaves angiotensinogen to (inactive) Ang I $\rightarrow$ ACE carboxypeptidase cleaves a single amino acid from (inactive) Ang I to generate (active) Ang II.

- (Active) Ang II upregulates ACE and downregulates ACE2. ${ }^{4}$

- Ang II inhibits renin.

- Ang II induces ACE2 shedding (facilitates loss of its negative regulator).

- Net effect: Increased Ang II $\rightarrow$ increased BP (positive feedback).

\section{ACE2 Protective Axis}

ACE2 is a key negative regulator of RAS, metabolizing Ang II to Ang 1-7.5

- Renin cleaves angiotensinogen to (inactive) Ang I $\rightarrow$ ACE2 carboxypeptidase removes 2 amino acids from active Ang II to generate (vasodilatory) Ang 1-7 $\rightarrow$ increased Ang 1-7 and decreased Ang $\| \rightarrow$ decreased BP.

- ACE2 limits vasoconstriction by removing Ang II.

- ACE2 limits vasoconstriction of Ang II via vasodilatory action of Ang 1-7.

- Ang 1-7 acting via the Mas receptor is cardioprotective.

- Net effect: Increased ACE2 $\rightarrow$ decreased BP (negative feedback).

- ACE2 acts as a counter-regulator to ACE, modulating the balance of vasoconstriction and vasodilation.

\section{What Controls ACE vs ACE2 Balance (Yin-Yang)?}

- In normal homeostasis, the ACE positive feedback loop provides amplification but with instability; the ACE2 negative feedback loop provides modulation, balance, and fine tuning. ${ }^{6}$

- High-risk demographic groups have ACE2 deficiency.

- SARS-CoV-2 infection; viral entry via ACE2 downregulates ACE2.

- Downregulation of ACE2 leads to overstimulation of the RAS. ${ }^{3}$

- Unopposed ACE and Ang II lead to predominance of the adverse axis and loss of control.

\section{SARS-CoV-2 infection leads to a shift in balance from the ACE2 protective axis to the ACE adverse axis with a loss of control.}

- Results in increased Ang II and its downstream actions via the AT, receptor, resulting in increased vasoconstriction, inflammation, and thromboses.

- These in turn develop into the clinical sequelae of COVID-19: ARDS, thromboses, MIS-C, cytokine storm, etc.

Abbreviations: ACE, angiotensin-converting enzyme; Ang, angiotensin; ARDS, acute respiratory distress syndrome; BP, blood pressure; MIS-C, multisystem inflammatory syndrome in children; RAS, renin-angiotensin system.

\section{RAS, ACE2, AND BP HOMEOSTASIS}

The RAS comprises several feedback loops maintaining BP homeostasis; ACE2 is central to this system (Table 1). In normal functioning, the ACE positive feedback loop provides amplification of angiotensin (Ang) II for a rapid response, while the ACE2 negative feedback loop modulates the ACE loop with smooth fine control of BP.

Inactive prorenin is produced by the juxtaglomerular cells in the kidney. Activated by decreased BP, the juxtaglomerular cells cleave prorenin to renin, which in turn cleaves angiotensinogen in the liver to (inactive) Ang I. Ang I is then converted to active Ang II by ACE in vascular endothelium cells of the lungs and kidneys. Ang II increases sodium reabsorption, increasing BP, ${ }^{7}$ thus forming a positive feedback loop.

ACE2 is a homologue of ACE. ACE2 is a zinc metalloenzyme expressed on the surface of endothelial cells and other cells.
ACE2 has 3 sections, an N-terminal signal sequence, a hydrophobic transmembrane, and a cytoplasmic section. ${ }^{8}$ ACE2 is multifunctional and can act on other systems. It is a chimeric protein thought to have emerged from gene duplication of ACE at the carboxypeptidase end and duplication of collectin at its transmembrane end. ${ }^{9}$ ACE2 receptors are found in the heart, vessels, the gastrointestinal tract, and the lungs. Approximately 83\% of ACE2-expressing cells are alveolar epithelial type II cells. $^{10}$

Both ACE and ACE2 are zinc enzymes. The major difference between ACE and ACE2 is the specificity of their carboxypeptidases - the ACE carboxypeptidase removes a single amino acid, while the ACE2 carboxypeptidase removes two amino acids. ${ }^{5}$ Because of this specificity, ACE converts inactive Ang I to active Ang II, which acts via the AT1 receptor with proinflammatory actions. Increased Ang II results in increased BP. 


\begin{tabular}{|c|c|c|c|c|c|}
\hline System & \multicolumn{3}{|c|}{ Activator/Feedback (Positive/Negative) } & Result & Clinical \\
\hline \multirow[t]{2}{*}{ RAS, ACE } & $\downarrow$ BP (positive) & Renin (positive) & ACE (positive) & \multirow{2}{*}{$\begin{array}{l}\uparrow \text { vasoconstriction, } \\
\text { inflammation, } \\
\text { thrombosis }\end{array}$} & \multirow{2}{*}{$\begin{array}{l}\text { ARDS, thrombosis, } \\
\text { MIS-C, cytokine } \\
\text { storm }\end{array}$} \\
\hline & $\begin{array}{l}\text { Prorenin to renin, } \\
\text { adverse axis }\end{array}$ & $\begin{array}{l}\text { Angiotensinogen to } \\
\text { (inactive) Ang I }\end{array}$ & $\begin{array}{l}\text { Ang I to (active) } \\
\text { Ang II }\end{array}$ & & \\
\hline \multirow[t]{2}{*}{ ACE2 } & & ACE2 (negative) & & \multirow{2}{*}{$\begin{array}{l}\text { Negative regulator } \\
\text { to ACE }\end{array}$} & \\
\hline & Protective axis & Circulating Ang II & & & \\
\hline $\begin{array}{l}\text { ACE2 chronic } \\
\text { deficiency }\end{array}$ & $\begin{array}{l}\text { Decreased ACE2 } \\
\text { in men, aged, } \\
\text { chronic disease }\end{array}$ & Viral entry & $\begin{array}{l}\text { Further } \\
\text { downregulates } \\
\text { ACE2 }\end{array}$ & $\begin{array}{l}\text { Downregulation of } \\
\text { ACE2 }\end{array}$ & $\begin{array}{l}\text { Disease progres- } \\
\text { sion in demo- } \\
\text { graphic groups }\end{array}$ \\
\hline \multirow{3}{*}{$\begin{array}{l}\text { Innate immune } \\
\text { system } \\
\text { (complement) }\end{array}$} & Classical & C1 & \multirow[t]{3}{*}{ Dysregulation } & \multirow{3}{*}{$\begin{array}{l}\text { Interferons, } \\
\text { cytokines }\end{array}$} & \multirow{3}{*}{$\begin{array}{l}\text { Acute exacerbation } \\
\text { of disease at days } \\
10-14, \text { ARDS }\end{array}$} \\
\hline & $\begin{array}{l}\text { Mannose-binding } \\
\text { lectin }\end{array}$ & $\begin{array}{l}\text { Mannose-binding } \\
\text { lectin }\end{array}$ & & & \\
\hline & Alternative & C3 & & & \\
\hline $\begin{array}{l}\text { Intestinal amino } \\
\text { acid homeostasis }\end{array}$ & $\begin{array}{l}\text { Amino acid defi- } \\
\text { ciency }\end{array}$ & Decreased ACE2 & $\begin{array}{l}\text { Viral entry further } \\
\text { downregulates } \\
\text { ACE2 }\end{array}$ & $\begin{array}{l}\text { Intestinal } \\
\text { inflammation }\end{array}$ & $\begin{array}{l}\uparrow \text { risk of disease } \\
\text { progression }\end{array}$ \\
\hline Complement & & & & $\begin{array}{l}\text { Activation of C3 } \\
\text { exacerbates ARDS }\end{array}$ & $\begin{array}{l}\text { Thrombosis, } \\
\text { ARDS, MIS-C }\end{array}$ \\
\hline
\end{tabular}

Abbreviations: ACE, angiotensin-converting enzyme; Ang, angiotensin; ARDS, acute respiratory distress syndrome; BP, blood pressure; MIS-C, multisystem inflammatory disease in children; RAS, renin-angiotensin system.

The major substrate for ACE2 is Ang II. ACE2 degrades (active) Ang II to generate (vasodilatory) Ang 1-7, resulting in decreased BP, thereby negatively regulating the RAS. ${ }^{5,9,11}$ The ACE2-Ang 1-7 axis works via the Mas receptor to mitigate the proinflammatory effects of the ACE axis. The ACE and ACE2 systems can thus be considered to have an "adverse" arm (ACEAng II-AT 1 receptor), and a "protective" arm (ACE2-Ang 1-7-Mas receptor).

\section{ACE2 DYSREGULATION AND CLINICAL SEQUELAE}

In normal functioning, the ACE positive feedback loop leads to amplification of Ang II and increased BP, while the ACE2 negative feedback loop modulates the ACE loop with smooth fine control (Table 1). ${ }^{6}$

Changes in ACE2 activity can alter the balance between the adverse and protective axes. Downregulation of ACE2 can lead to over-stimulation of the RAS and increase in Ang II; acting via the $\mathrm{AT}_{1}$ receptor to increase vasoconstriction, inflammation and thromboses.

Preexisting ACE2 deficiency coupled with downregulation of ACE2 associated with viral entry can result in an imbalance of the "yin and yang," 12 with predominance of the adverse axis, resulting in complete loss of control. ${ }^{13}$ This loss of control can be signaled downstream via the $\mathrm{AT}_{1}$ receptor, with dysregulation and devastating downstream consequences. ${ }^{13,14}$ Reciprocal inhibitory pathways, in certain situations (eg, hypoxia), can form positive feedback loops that can shift the balance between the adverse and protective axes. ${ }^{12}$

\section{ACE2 AND VIRUS ENTRY INTO EPITHELIAL CELLS}

"Surreptitiously," SARS-CoV-2 also utilizes ACE2 as a cellular entry receptor. ${ }^{6}$ It is not known why this key enzyme of the RAS serves as the entry point for SARS viruses; the answer may lie in the distant phylogenetic past. In invertebrates as well as vertebrates, ACE2 interacts with the innate immune system responsible for the rapid nonspecific response to an invading pathogen. ACE interacts with macrophages and neutrophils, increasing their antibacterial function, especially if they overexpress ACE..$^{15}$

To enter cells, the viral spike glycoprotein $S$ binds to the host cell ACE2 receptor. ${ }^{16,17}$ This is followed by cleavage by cathepsin, activation of a fusion peptide, and release of the viral genome into the cell. This is accompanied by downregulation of the ACE2 receptor.

\section{DEMOGRAPHIC GROUPS AND COVID-19}

Persons older than 65, men, and those of minority race or ethnicity are at higher risk of contracting SARS-CoV-2, and 


\begin{tabular}{l} 
Table 3. Selected Medications That May Affect ACE, ACE2, or Other \\
Parts of the RAS \\
\hline ACEIs $^{35}$ \\
\hline Benazepril \\
\hline Captopril \\
\hline Enalapril \\
\hline ARBs \\
\hline Azilsartan \\
\hline Candesartan \\
\hline Zinc Chelators/Zinc Supplementation \\
\hline Zinc 33,36 \\
\hline ACE2 \\
\hline Recombinant ACE2 \\
\hline Other Drugs That May Upregulate ACE2 \\
\hline Tretinoin ${ }^{37}$ \\
\hline Thiazolidines \\
\hline Ibuprofen \\
\hline
\end{tabular}

once infected are at higher risk of progression of COVID-19 and death. These same groups are also associated with increased rates of hypertension. Persons with hypertension in these groups have more rapid progression to severe hypertension with increased incidence of stroke, cardiovascular disease, and kidney damage. These same demographic factors also are associated with ACE2 deficiency.

It has been known since the first cases in Wuhan that COVID-19 attacks men more than women, and that patients older than 65 years or with underlying chronic conditions such as obesity, hypertension, of diabetes, have a poorer prognosis. Conti and colleagues ${ }^{18}$ propose that aging results from chronic activation of RAS due to increased production of tissue reactive oxygen species, which is increased by Ang II.

As newer analyses of COVID-19 data are including demographics by race and ethnicity, a consistent pattern is emerging. African Americans, Hispanics, and indigenous populations are being affected disproportionally with higher infection rates, higher rates of progression to severe disease, and higher mortality. The Centers for Disease Control and Prevention (CDC) has reported that COVID-19-related deaths per 100,000 population were double for Blacks (92.3) compared with Whites (45.2). ${ }^{19}$ They summarized that, compared with White non-Hispanics, Blacks or African Americans have a 2.6-fold higher number of cases, a 4.7-fold higher rate of hospitalization, and a 2.1-fold higher death rate. ${ }^{19}$ The $\mathrm{CDC}$ also has reported that 8 of 10
COVID-19-related deaths were in patients older than 65 years. ${ }^{20}$

In the United Kingdom, Black women are 4.3 times more likely, and Black men 4.2 times more likely, to die from COVID-19 compared with their White counterparts. ${ }^{21}$ Persons of Bangladeshi, Indian, or Pakistani descent were also statistically more likely to die compared with Whites. ${ }^{21}$

\section{DEMOGRAPHIC GROUPS AND HYPERTENSION}

It has long been appreciated that men are more likely to have hypertension, for that hypertension to start at an earlier age, for it to progress more rapidly, and for it to be associated with longterm poor outcomes (eg, cardiovascular disease, stroke).

The baseline characteristics associated with higher rates of incidence and progression of hypertension are virtually identical to those with higher rates of incidence, progression, and death with COVID-19: advanced age, male sex, African American or other minority group, and underlying chronic disease (eg, hypertension, diabetes, obesity). Given these similarities, is there a plausible biological mechanism that would explain how these socioeconomic factors could result in increased incidence and progression of hypertension and increased infection with SARSCoV-2 and progression of COVID-19?

\section{ACE2 DEFICIENCY}

ACE2 serves as the viral entranceway to the cell. ${ }^{6}$ ACE2 deficiency has been hypothesized to explain the demographic risk factors for SARS-CoV-2 infection, with further downregulation of ACE2 associated with viral infection. ${ }^{6}$ ACE2 deficiency has been noted in the following subgroups: those with advanced age; men; and those with diabetes, hypertension, or chronic lung disease. Individuals in each of these subgroups have also experienced higher rates of infection and higher progression of disease and death.

Verdecchia and colleagues ${ }^{6}$ noted that the groups with higher rates of disease progression with COVID-19 have been noted to also have higher rates of ACE2 deficiency. Thus, ACE2 deficiency has been documented with aging, more so in men than in women, in diabetes, and in exacerbation of hypertension. Indeed, several studies suggest that ACE2 deficiency is a causative factor in hypertension. Chronic ACE2 deficiency in those patients with hypertension or increased incidence/progression of COVID-19 may suggest a correlation rather than a cause-effect relationship.

High blood levels of ACE2. Sama and Voors have found that men have higher blood levels of ACE2 than women. ${ }^{22}$ They point out that the ACE2 gene is located on the X chromosome, and that X-linked diseases are generally more severe in men than in women. However, ACE2 is predominantly bound to cell membranes in heart, kidneys, and testes, while soluble ACE2 is only scarcely found in the circulation. Soluble ACE2 is shed from the membrane-bound form by the ADAM17 enzyme. ${ }^{22}$ Coexpression of these in the testes might help explain why blood 
levels of ACE2 are higher in men than in women. ${ }^{22}$ The importance of these blood levels and the more severe course of COVID-19 in men needs further investigation.

Sama and Voors also noted that ACE inhibitors (ACEIs) or angiotensin-receptor blockers (ARBs) do not increase ACE2 levels, and this suggests that their continued use is safe in patients who become infected with SARS-CoV-2.

Tikellis and Thomas ${ }^{23}$ have reviewed the actions of ACE2, not only in its moderating action in the RAS, but also its beneficial actions in atherosclerosis, hypertension, heart failure, chronic kidney disease, and in the lung. Many of these direct actions are modest; however, in the setting of RAS activation, if ACE2 is deficient, the negative results are much more intense.

\section{ADDITIONAL FACTORS POSSIBLY IMPACTING ACE2}

Smoking. Smoking has been associated with an increase in ACE2, particularly in pulmonary goblet cells. ${ }^{24}$

Amino acids and intestinal inflammation. Hashimoto and colleagues $^{25}$ discovered the importance of ACE2 in amino acid transport. When amino acid transport is disrupted, intestinal inflammation is increased. The intestinal inflammation might explain the high-risk conditions, but what causes the inflammation? Could it be a history of other coronavirus infections?

History of prior coronavirus infections. Another link may be a history of infection with other coronaviruses, including the common cold. Perhaps individuals with hypertension, diabetes, older age, and male sex have been exposed to coronaviruses, and their spike glycoprotein protein $S$ has bound to ACE2, leading to viral cell entry and downregulation of the ACE2 receptor. ${ }^{6}$

Qian and colleagues ${ }^{26}$ noted unexpected high interpatient variability of ACE2. This variability may reflect the above covariates and perhaps be associated with different stages of disease and response along the transition from "protective" to "adverse" axes (Tables $\mathbf{1}$ and $\mathbf{2}$ ).

\section{AGING AND THE RAS}

How does age manifest, and why does advanced age get recognized and become a baseline characteristic associated with a high risk of disease progression and death?

"Inflamm-aging" and immune senescence. Bonafe and colleagues ${ }^{27}$ emphasize that aging, as can be measured by telomere shortening, is characterized by what they call "inflamm-aging" (chronic subclinical systemic inflammation) and immune senescence. They hypothesize that the inflammation and immune senescence of aging result in a downregulation of ACE2. In addition, elevated levels of interleukin 6, which are typical of aging, promote lung inflammation, leading to cytokine storm. Bonafe and colleagues also point out that this chronic inflammation, although characteristic of older men, may also occur in others, resulting in higher susceptibility to SARS-CoV-2 infection.

The association of ACE2 deficiency in diabetic glycoxidation has led to proposing the therapeutic use of ACE2 for diabetic kidney disease ${ }^{28}$ favoring the "preserved" ACE2 axis over the "adverse" ACE axis.

Surfactant. A clinical trial of a surfactant and a nebulizer device has been proposed for the treatment of COVID-19. ${ }^{29}$

\section{SOCIOECONOMIC FACTORS AND RISKS \\ WITH COVID-19 AND HYPERTENSION}

A recent opinion ${ }^{30}$ emphasized the racial inequality in COVID-19, with African Americans being more likely to work in service jobs, putting them at higher risk of being exposed to the virus; living in substandard housing in communities impacted by alcohol, tobacco, and drugs; and unequal access to health infrastructure. All these factors certainly contribute to the overrepresentation in progression of and deaths from COVID-19. What is unclear is how these social and economic factors might impact the individual or the pathophysiology of infection, resulting in a higher incidence of disease, more-severe disease, and increased disease progression to death. It can be hypothesized that these socioeconomic factors could impact the individual as chronic conditions resulting in deficiency of ACE2. As noted above, data are accumulating that suggest that those with ACE2 deficiency have a higher incidence of COVID-19 and poorer prognosis once infected.

\section{ACE AND INDIRECT LINKS TO THE INNATE IMMUNE RESPONSE}

The innate immune system refers to the initial nonspecific response to a pathogen via primitive mechanisms shared across species. The response uses components of the complement system via 3 pathways - classical, mannose-binding lectin, and alternative-to trigger different substrates in the complement cascade via positive feedback loops to in turn release cytokines and interferons. ${ }^{31,32}$

The time-course of exacerbation of COVID-19 at approximately days 10 to 14 could represent an aberrant immune response, with overstimulation of the innate response or inadequate initiation of the pathogen-specific adaptive immune response.

Although ACE and ACE2 are not direct factors in the pathways of the innate immune system, ACE can cleave other peptides besides Ang I and thus can have a number of actions independent of the RAS. ${ }^{31} \mathrm{ACE}$ can modulate macrophage and neutrophil actions, thus possibly impacting both the innate and adaptive immune responses.

Aberrant or dysfunctional overstimulation of the innate immune response can lead to cytokine storm or acute respiratory distress syndrome (ARDS), and perhaps multisystem inflammatory syndrome in children (MIS-C), which has recently been reported in children infected with SARS-CoV-2.

These aspects of ACE2 can help explain many of the clinical manifestations of SARS-CoV-2 infection and COVID-19 and may offer insight into treatment options to control this pandemic. 


\section{CHANGES IN ACE2 EXPRESSION WITH MEDICATIONS}

Given the dysregulation of the RAS that can occur in COVID-19 (Tables 1 and 2), Zamai ${ }^{12}$ has suggested the use of ACEIs and ACE2 inhibitors as a means of preventing the effects of feedback loop imbalance. Inhibition could be at the level of ACE2 enzyme, with zinc-chelating drugs inhibiting the ACE and ACE2 zinc metalloenzyme, or downstream with renin inhibitors; the Food and Drug Administration has approved drugs in both categories. A randomized clinical trial of zinc supplementation has been initiated by the Cleveland Clinic. ${ }^{33}$ The use of ACEIs has sometimes been avoided in African American persons due to the perception that their efficacy is lower in this population. ${ }^{34}$

Table 3 lists selected medications that may affect ACE, ACE2, or other parts of the RAS. (Note that drugs that are usually considered agonists are listed in the same table as those that are considered antagonists. In feedback systems, a perturbation of intensity or timing that is beyond the limits of the system can result in a usually negative feedback acting as a positive feedback, resulting in disruption and failure of the feedback system. Thus, an intervention that is beneficial at one point may be harmful at a different point.)

Disruption of the normal feedback loops or decreased activity of ACE2 can lead to paradoxical increases in Ang II and aldosterone with the use of RAS inhibitors. Exaggerated feedback response resulting in hypertension in patients on RAS inhibitors can contribute to cardiac, vascular, and renal damage. ${ }^{12,13}$ (For a more detailed discussion see Zamai ${ }^{12}$ ).

Should ACEIs be continued or discontinued in patients who are taking them for hypertension and who are infected with SARS-CoV-2? Diabetes and hypertension are among the most frequently encountered comorbidities seen in those hospitalized for COVID-19. Fang and colleagues ${ }^{16}$ pointed out that with ACEIs and ARBs in diabetes, and with ACEIs in hypertension, ACE2 expression is increased. ACEIs, thiazolidines, and ibuprofen can also upregulate ACE2. ${ }^{16}$ Pathogenic coronaviruses bind to epithelial cells in lung, intestine, kidney, and blood vessels. ${ }^{38}$ Fang and colleagues hypothesize that treatment with these drugs that increase expression of ACE2 may increase the risk of progression to severe and fatal COVID-19. ${ }^{16}$ Thus, monitoring for these drugs and substituting others not associated with increases in ACE2 might decrease the progression to severe disease and death in these patients.

In a letter to medical professionals, GSK Consumer Healthcare pointed out that these hypotheses were presented without any supportive clinical data. ${ }^{39}$ The company also pointed out that hypotheses such as these need to be addressed with randomized controlled studies.

A retrospective observational (nonrandomized) study used propensity-scored analyses comparing 1128 hypertensive patients diagnosed with COVID-19, 188 (16.7\%) of whom were taking ACEIs or ARBs and 940 (83.3\%) of whom were not taking ACEIs or ARBs. ${ }^{40}$ This study showed a decreased all-cause mortality in those taking the ACEIs or ARBs, with 7 of 188 (3.7\%) deaths on ACEIs or ARBs and 92 of 940 (9.8\%) in those not taking ACEIs or ARBs. The authors concluded that it was unlikely that the use of ACEIs or ARBs increased all-cause mortality in those taking them.

Magrone and colleagues ${ }^{41}$ have similarly suggested that ACE2, since it binds to coronaviruses, should be considered as a therapeutic target. Binding of the viral S1 protein to ACE2 is required for viral entry and also leads to downregulation of ACE2. This in turn leads to increased Ang II by ACE acting on the AT receptor. Batlle and colleagues have proposed use of recombinant ACE2 for treatment of COVID-19 and its complications. ${ }^{42}$ Coronavirus infection can inhibit production of interferon; this may be via the complement pathways. Magrone and colleagues also propose exploring the use of polyphenols, including resveratrol, which can increase ACE2 levels. ${ }^{41}$

In a note added in proof, Magrone and colleagues ${ }^{41}$ (citing Hanff and colleagues ${ }^{43}$ ) attribute the cardiovascular mortality of COVID-19 to dysregulation of the RAS.

\section{OTHER FEEDBACK SYSTEMS AND COVID-19}

Complement and viruses. The complex complement pathways (including lectin, classical and alternate pathways, and multiple feedback loops) do not include ACE2 per se, however complement activation is thought to contribute to the severity of ARDS seen with coronavirus infections. ${ }^{44}$ Complement C3-deficient mice infected with SARS-CoV-2 were shown to have less weight loss, less respiratory dysfunction, and less inflammation in the lungs compared with control mice. The authors suggested that inhibiting complement activation might be beneficial but pointed out the importance of complement system in reacting to bacterial and other pathogens. Compounds that block $\mathrm{C} 3$ are being tested in COVID-19 patients. ${ }^{32} \mathrm{C} 5$ inhibitors have proven safe and might be used in ARDS, known to be impacted by the $\mathrm{C} 5 \mathrm{a}-\mathrm{C} 5 \mathrm{aR}$ axis. Other branches $(\mathrm{C} 3 \mathrm{a}-\mathrm{C} 3 \mathrm{aR})$ might limit these reactions. ${ }^{32}$

Complement systems pathways are also involved with the pathogenesis of COVID-19-associated ARDS; activation of C3 exacerbates ARDS. Janeway and colleagues offer an excellent review of the complement system. ${ }^{45}$ For an overview of complement and potential touchpoints with COVID-19 and ARDS, see articles by Gralinski and colleagues, ${ }^{44}$ Stoermer and colleagues, ${ }^{46}$ and Risitano and colleagues. ${ }^{47}$ The complement-ARDS links are beyond the scope of this review.

$A C E s$ in reproduction. Intriguingly, it has long been known that semen is high in zinc, that zinc deficiency is associated with sterility, and that anosmia and ageusia are symptoms of zinc 
deficiency. ${ }^{48,49}$ Anosmia and ageusia are considered early symptoms of COVID-19, suggesting a possible link.

Both ACE1 and ACE2 are required for normal female development and function, and ACE2 is required for normal male development and function. ${ }^{50}$ ACE1 acts via converting Ang I to Ang II and the $\mathrm{AT}_{1}$ and $\mathrm{AT}_{2}$ receptors in female reproduction. In males, $\mathrm{ACE} 2$ and the $\mathrm{AT}_{2}$ receptor are needed for reproductive function. A third ACE, ACE3, is active in the testes of mice, bulls, and dogs, but not in humans.

Hagaman and colleagues ${ }^{51}$ have used genetic targeting in mice to further explore the functions of ACEs, including the somatic isozyme ACE and the testicular isozyme tACE. Male ACE homozygous mutants have reduced fertility, while female ACE homozygous mutants are fertile. Thus, missing somatic ACE does not halt fertility, and tACE is sufficient for normal fertility. The functions of ACE and tACE in male reproduction remain unknown.

\section{EVOLUTION}

A number of ACE isoforms are present in mammals but also in insects and leeches. ACE is found in multiple species (fish, amphibians, and mammals, ${ }^{52}$ as well as mosquitoes and sea squirts). ${ }^{15}$ In mammals, ACE activation increases vasoactive Ang II, while ACE2 has an antagonistic role, serving to moderate ACE vasoactive response. The testicular isoform is needed for male fertility. ACEs have been found in insects despite their lack of a closed circulatory system. An active ACE has also been found in the digestive tract of leeches. These findings suggest a long evolutionary history of ACEs with unknown function but providing a survival advantage. The role of ACEs in the innate immune response, coupled with their use by viruses for cell entry, remind us how little we know.

The evolution of ACE and ACE2 and their isoforms suggests that natural selection may be acting at the "module" level rather than at a single gene level. The genetic duplication of carboxypeptidase and collectin, for example, might provide a transfer of a several genes working together as a functional module. Interestingly, engineers working with feedback systems can easily change modules-for example, switching an analogue sensor to a digital sensor. Although such a switch might produce the desired output, the time delay may be longer, which may lead to instability of the new system.

Another consideration in regard to natural selection: One might expect a component or module from a feedback system to be more likely to be available for transmission to the next generation if the positive and negative feedback loops are in balance and allow some oscillations. Put another way, if a system has only a positive feedback loop, then it will be unstable and fail, while if only a negative feedback loop is present, the system will remain stagnant. For selective pressure (or any force for change) to act, it needs something on which to act. That something is only there if the positive and negative feedbacks are in balance, not if stagnant or failed. ${ }^{14}$ Conservation of molecular structure across phylogenetic branches suggests that the sequence had or has an important functions, ${ }^{52}$ which may be unrelated to its function in mammals and which may remain elusive to us. Prolactin and related growth hormone, for example, are thought to have expanded, with their receptor family, by duplication, with the various forms filling functions as diverse as milk production, development, and metabolism. ${ }^{53}$ In bony fish, prolactin regulates osmoregulation. Other functions found in a variety of species include growth and development, metabolism, reproduction and immunoregulation. ${ }^{54}$

\section{WHY BATS?}

Bats are the reservoir host for a number of zoonoses including Ebola virus disease, Marburg hemorrhagic fever, rabies, and Nipah virus disease. While these viruses are devastating to other mammals, including humans, infected bats have no symptoms. In most mammals, the innate immune response responds to viral invasion by initiating nonspecific inflammatory reactions, including the production of interferon, initiating an intense immune reaction. In bats, however this interferon pathway is always on; the bats are able to limit the damage that an "always on" interferon pathway would cause by other anti-inflammatory mechanisms. This "always on" control of the interferon pathway can select for high viral prorogation. Thus, bats can tolerate and control highly propagating viruses without the intense inflammatory response that characterizes infection in other species and leads to the morbidity and mortality seen in infection..$^{55,56}$

\section{FEEDBACK SYSTEMS}

So systems science is not a study of 'things'; it is about how things are connected. Systems scientists set out to study the properties associated with such 'connectedness.' One consequence of this is that, if elements are connected in the same way, they will behave in the same way, irrespective of their material form. So knowledge gleaned from the study of physical systems (eg, molecules, organisms) can be applied to social (eg, economics) and even logical systems.

From Future Ready: How to Master Business Forecasting ${ }^{14}$

A stable negative feedback system can change to act as an unstable positive feedback loop if the timing of response exceeds the rate of disturbance for which the system was designed. ${ }^{14}$ (For an introduction to feedback loops, see references 2, 58, 59, and 64.) In the mid 1800s, J.C. Maxwell realized that this could explain a major contemporary issue-why steam locomotives with centrifugal governors had a tendency to explode. ${ }^{57}$

Steam engines have governors that are designed to release the 


\section{Angiotensin-Converting Enzyme 2, Feedback Systems, and COVID-19}

\section{1}

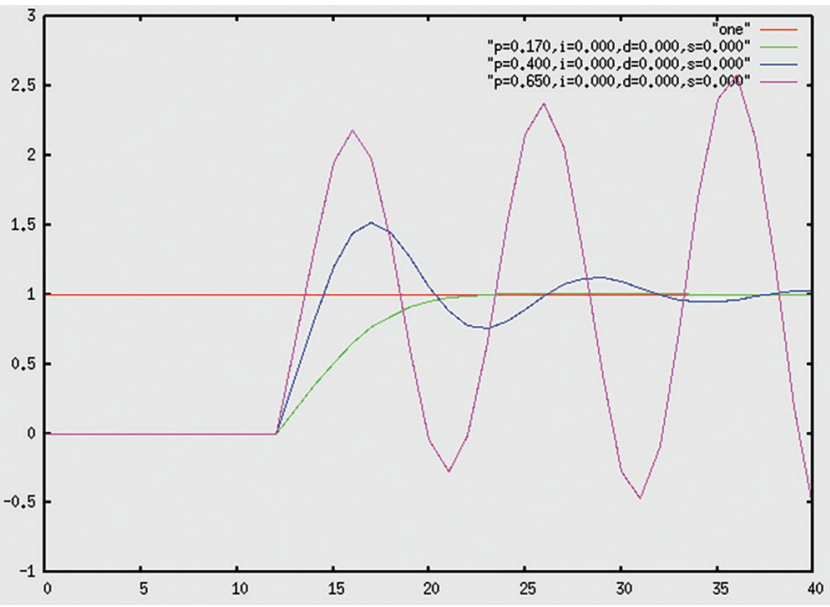

This graph demonstrates what happens if the constant $k_{p}$ (proportional gain) is too large (greater than that for which the system was designed). ${ }^{58}$ Reproduced with permission John Wiley \& Sons.

throttle if the speed exceeds a set level. A simple Watt governor consists of weights on hinged arms rotating on an axle geared to the speed. When rotating above a set speed, the weights move outwards via centrifugal force and move a lever releasing the throttle (a simple negative feedback loop). Early steam engines were stationary, used for pumps, mines, or factory power; perturbations were small, and the governors worked well under the designed conditions. Later, when steam engines were used on locomotives, the perturbations affecting the governors were larger. If there is a perturbation greater than the designed limits of the governor, or the timing of the governor signal is delayed (eg, by friction), the usual negative feedback can act as a positive feedback and lead to increasingly wide oscillations and failure.

Maxwell used differential equations and then solved them to describe components of disturbances to the governor-controlled system. ${ }^{57}$ These components may be of 4 different kinds:

1. The disturbance may continually increase.

2. It may continually diminish.

3. It may be an oscillation of continually increasing amplitude.

4. It may be an oscillation of continually decreasing amplitude.

The first and third cases are evidently inconsistent with the stability of the motion, and the second and fourth alone are admissible in a good governor. Keeping the accuracy and parameters of the governor within preset limits, dysregulation (cases 1 and 3) could be avoided.

Hellerstein and colleagues ${ }^{58,59}$ created response curves using

\section{2}

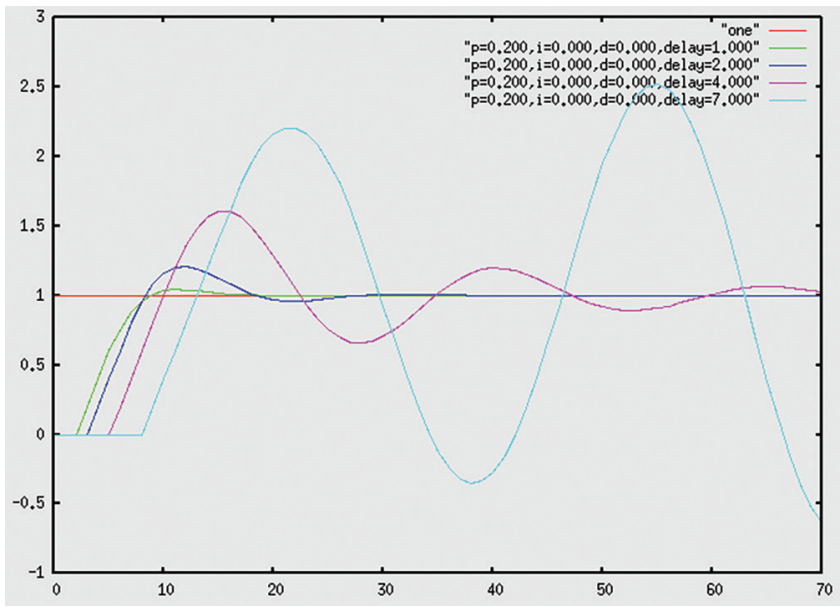

This graph demonstrates what happens if the time of action of the feedback signal is delayed. ${ }^{58}$ Reproduced with permission John Wiley \& Sons.

a computer simulator using the programming language Python and graphed with the graphing utility Gnuplot. Their PID model (proportional, integral, and derivative) had as input the sum of a constant multiplied by the proportion, a second constant multiplied by the integral, and a third constant multiplied by the derivative of the error. (PID control is also covered by Åström and Murray. ${ }^{2}$ )

The PID controller:

\section{control_input $=k_{p}$ control_error $+k_{i} \int$ control_error $+k_{d} d / d t$} control_error

The three components reflect the past (integral, area under the curve up to time $t$ ), the present (proportional, at time $t$ ) and the prediction of the future (differential, based on rate of change at time $\mathrm{t})^{2}$

Figure 1 demonstrates what happens if the constant $k_{p}$ (proportional gain) is too large (greater than that for which the system was designed). Figure $\mathbf{2}$ demonstrates what happens if the time of action of the feedback signal is delayed. In these situations, if the perturbation is too large, or the signal sufficiently delayed, the negative feedback can act as a positive feedback, leading to oscillations of increasing amplitude and loss of control. The simplest model had constants for the integral and derivatives set to zero; thus, the input is only proportional to the error. ${ }^{58}$ 
In Figure 1, the 3 lines differ only by the constant to which the proportion is multiplied. The proportional gain (with different slopes seen at $\mathrm{Y}=0$ ), produces curves with constant too high (red; oscillations increasing in amplitude), too low (green; slow to respond), and "just right" (blue; oscillations decreasing in amplitude). With Maxwell's steam engines, perturbations increased when the steam engines were no longer stationary but on a locomotive.

Figure 2 shows results with inputs only differing in the amount of time delay in the control loop, with delays of 1 (green; good), 2 (dark blue; overshoot worse, slower to respond), 4 (magenta; barely stable) and 7 (light blue; unstable with oscillations increasing in amplitude). The differences in the time delay can be seen at $\mathrm{Y}=0$. Time delays can come from a variety of sources; in Maxwell's locomotives, friction within the governor was a main contributor.

These are examples of how a stable negative feedback homeostatic system can change to an unstable response and failure if the perturbations are too large or the time delays too long. However, if bounds can be set for the input and output such that the system remains stable (described as a bounded-input, bounded-output [BIBO] stability), ${ }^{60}$ dysregulation can be avoided.

If you find that a given input gives you dramatically different outputs at different times or circumstances, you are likely dealing with a feedback loop. For example, as Tikellis and Thomas noted, ${ }^{23}$ beneficial actions may result in modest effect; however, in the setting of RAS activation, if ACE2 is deficient, the negative results may be much more intense. Exaggerated feedback responses also can explain paradoxical increases in BP in many patients on RAS blockers.

Excessive or delayed activation of components of a natural homeostatic mechanism may also underlie the pathophysiology of coronavirus-related morbidity: COVID-19-associated ARDS, cytokine storm, and MIS-C may all represent excessive activation of normally protective feedback loops.

\section{MATHEMATICAL MODELS}

A number of investigators have developed mathematical models for the RAS system or its components. Computational models have been proposed and built, requiring extensive medical, scientific, and technical interdisciplinary cooperation.

Guillaud and Hannaert ${ }^{61}$ created a computer model of renin and circulating RAS, based on Guyton's circulatory model and a variety of other sources and toolboxes. The model input included data from selected data sets. The model produced realistic responses to BP homeostasis and in the future may be useful for exploring pharmacotherapeutics. Barbiero and Lió ${ }^{62}$ have developed a model with the descriptive title, "The computational patient has diabetes and a COVID."
Others have focused in on isolated segments within the RAS system, comparing specific inputs and dynamic outputs. Koka and colleagues ${ }^{4}$ studied tissue from patients without or with hypertensive cardiomyopathy or hypertensive nephropathy. In the nonhypertensive tissue, ACE expression was high while in hypertensive tissue, inhibiting ACE2 led to ACE upregulation and ACE2 downregulation. Ang II is thought to downregulate the ACE2 in hypertension via the $\mathrm{AT}_{1}$ receptor pathway. This ACE/ACE2 imbalance is implicated in hypertensive cardiovascular or renal damage.

\section{TIME COURSE OF COVID-19 AND CREATING A MODEL}

Summaries of the clinical progression of COVID-19 often note that many patients do relatively well in the first week after onset but then may experience an abrupt exacerbation, often with ARDS or other sequelae in the second week. For example, Liu J and colleagues ${ }^{63}$ reviewed the clinical histories of 276 patients and selected 24 patients meeting predefined criteria of confirmed COVID-19 pneumonia. They noted clinical exacerbation (defined as moving from the general ward to the intensive care unit) at 8.8 days after disease onset. With exacerbation, patients had more clinical symptoms, and an increase in number of lung lobes involved, with multifocal lesions in 100\%.

In the context of control systems, we hypothesize that this abrupt exacerbation at the beginning of the second week of illness represents a "tipping point" with the onset of dysregulation, a shift from the "protective" to predominance of the "adverse" axis of the RAS system (Tables $\mathbf{1}$ and 2), oscillations increasing in amplitude, and loss of control. A corollary of this hypothesis is that early in infection (the first week), most patients would still have the protective axis predominant. Those who experience an exacerbation in the second week would have the adverse axis predominant and loss of control. This would have to be confirmed with clinical data. Although this shift is likely to be complex, we should be able to estimate input variables. Modeling a control system with these variables will allow us to identify conditions and derive limits that keep the system stable. ${ }^{58}$ The data-derived model would provide a rational basis for choice and timing of therapeutic interventions while avoiding those areas which might lead to dysregulation and unanticipated "paradoxical" negative outcomes.

Formal analysis and mathematical modeling of these feedback systems is bringing our understanding of COVID-19 pathophysiology to a higher level.

\section{CONCLUSION}

One enzyme, ACE2, is a key point of intersection for multiple control systems (renin-angiotensin, bradykinin) and indirectly linked to others (complement, innate immune response). ACE2 is also the major mechanism for viral entry into cells. 


\section{Angiotensin-Converting Enzyme 2, Feedback Systems, and COVID-19}

Thus, COVID-19 infection is intimately linked to these feedback systems of the host.

The RAS system includes positive (ACE) and negative (ACE2) feedback loops. Under normal (noninfected) conditions, the positive ACE feedback loop provides amplification of Ang II for a rapid response, while the negative ACE2 feedback loop modulates the positive and provides fine control of BP. The negative ACE2 "protective" axis may become weakened or diminished if there is preexisting ACE2 deficiency, as is the case with a number of demographic features, including age greater 65, male sex, and African American or another minority group. Superimposed on this preexisting ACE2 deficiency, SARS-CoV-2 infection and viral entry via ACE2 are associated with downregulation of ACE2. No longer constrained by the ACE2 negative protective control axis, the adverse ACE axis, and especially increased Ang II, overwhelms the RAS system, leading to increasing oscillations and loss of control. ${ }^{3}$

With this loss of control of the RAS, the increased Ang II can act on the usual downstream $\mathrm{AT}_{1}$ receptors with an increase in vasoconstriction, inflammation, and thromboses. These in turn lead to the major clinical sequelae of COVID-19, including ARDS, MIS-C, and cytokine storm. This failure of RAS control has been described as a loss of balance of the "yin and yang" of homeostasis. ${ }^{6,12}$ We can hypothesize that the clinical exacerbation often seen after the first week of COVID-19 may represent the onset of this "tipping point."

The ACE2 and RAS systems suggest a number of potential therapeutic interventions. However, these have to be approached and studied cautiously in the context of the feedback systems involved. A given intervention may have opposing outcomes depending on the status of the feedback systems (eg, whether the "protective" or "adverse" axis is predominant). Knowledge of the feedback systems will help us avoid potentially dangerous and unanticipated "paradoxical" outcomes.

\section{REFERENCES:}

1. Hoagland M, Dodson B, Hauck J. Exploring the Way Life Works: The Science of Biology. Jones and Bartlett Publishers; 1995:209.

2. Åström KJ, Murray RM. Feedback Systems: An Introduction for Scientists and Engineers. Princeton University Press; 2008:1.

3. Silhol F, Sarlon G, Deharo J-C, Vaïsse B. Downregulation of ACE2 induces overstimulation of the renin-angiotensin system in COVID-19: should we block the renin-angiotensin system? Hypertens Res. 2020;43(8):854-856. doi:10.1038/s41440-020-0476-3

4. Koka V, Huang XR, Chung ACK, Wang W, Truong LD, Lan HY. Angiotensin II up-regulates angiotensin I-converting enzyme (ACE), but down-regulates ACE2 via the AT1-ERK/p38 MAP kinase pathway. Am J Pathol. 2008;172(5):1174-1183. doi:10.2353/ajpath.2008.070762

5. Patel VB, Zhong JC, Grant MB, Oudit GY. Role of the ACE2/angiotensin 1-7 axis of the renin-angiotensin system in heart failure. Circ Res. 2016;118(8): 1313-1326. doi:10.1161/CIRCRESAHA.116.307708

6. Verdecchia P, Cavallini C, Spanevello A, Angeli F. The pivotal link between ACE2 deficiency and SARS-CoV-2 infection. Eur J Intern Med. 2020;76:1420. doi:10.1016/j.ejim.2020.04.037

7. Fountain JH, Lappin SL. Physiology, renin angiotensin system. StatPearls Updated July 27, 2020. Accessed September 29, 2020. https://www.ncbi. nlm.nih.gov/books/NBK470410/

8. Turner AJ, Hooper NM. Angiotensin-converting enzyme 2. In: Barrett AJ,
Rawlings ND, Woessner JF, eds. Handbook of Proteolytic Enzymes. Vol 1. 2nd ed. 2004:349-351. doi:10.1016/B978-0-12-079611-3.50092-6

9. Kuba K, Imai Y, Penninger JM. Multiple functions of angiotensin-converting enzyme 2 and its relevance in cardiovascular disease. Circ J. 2013;77(2): 301-308. doi:10.1253/circj.CJ-12-1544

10. Zhao Y, Zhao Z, Wang Y, Zhou Y, Ma Y, Zuo W. Single-cell RNA expression profiling of ACE2, the receptor of SARS-CoV-2. Am J Respir Crit Care Med. Published online July 14, 2020. doi:10.1164/rccm.202001-0179LE

11. Turner JT. ACE2 cell biology, regulation, and physiological functions. In: Unger T, Steckelings UM, dos Santos RAS, eds. The Protective Arm of the Renin Angiotensin System: Functional Aspects and Therapeutic Implications. Academic Press; 2015:185-189. doi:10.1016/B978-0-12-8013649.00025-0

12. Zamai L. The yin and yang of ACE/ACE2 pathways: the rationale for the use of renin-angiotensin system inhibitors in COVID-19 patients. Cells. 2020; 9(7):E1704. doi:10.3390/cells9071704

13. Thomas $M C$, Tikellis $C$, Losing control: positive and negative feedback in the renin angiotensin system. Curr Hypertens Rev. 2009;5(3):222-226. doi:10.2174/157340209788921194

14. Morlidge S, Player S. Appendix 2: important concepts in systems and cybernetics. In: Morlidge S, Player S. Future Ready: How to Master Business Forecasting. John Wiley \& Sons Ltd. 2010. Accessed August 25, 2020. https://onlinelibrary.wiley.com/doi/pdf/10.1002/9781119206613.app2

15. Bernstein KE, Khan Z, Giani JF, Cao D-Y, Bernstein EA, Shen XZ. Angiotensin-converting enzyme in innate and adaptive immunity. Nat Rev Nephrol. 2018;14(5):325-336. doi:10.1038/nrneph.2018.15

16. Fang L, Karakiulakis G, Roth M. Are patients with hypertension and diabetes mellitus at increased risk for COVID-19 infection? Lancet Respir Med. 2020; 8(4):e21. doi:10.1016/S2213-2600(20)30116-8. Published correction appears in Lancet Respir Med. 2020;8(6):e54.

17. Baden LR, Rubin EJ. Covid-19 - the search for effective therapy. N Engl J Med. 2020;382(19):1851-1852. doi:10.1056/NEJMe2005477

18. Conti S, Cassis P, Benigni A. Aging and the renin-angiotensin system. Hypertension. 2012;60(4):878-883. doi:10.1161/HYPERTENSIONAHA. 110.155895

19. Centers for Disease Control and Prevention. Coronavirus disease 2019 (COVID-19): COVID-19 hospitalization and death by race/ethnicity. Updated August 18, 2020. Accessed September 29, 2020. https://www.cdc.gov/ coronavirus/2019-ncov/covid-data/investigations-discovery/hospitalizationdeath-by-race-ethnicity.html

20. Centers for Disease Control and Prevention, Coronavirus disease 2019 (COVID-19): older adults. Updated September 11, 2020. Accessed September 29, 2020. https://www.cdc.gov/coronavirus/2019-ncov/need-extraprecautions/older-adults.html

21. Picheta R. Black people in the UK four times more likely to die from Covid 19 than white people, new data shows. CNN. May 7, 2020. Accessed September 29, 2020. https://www.cnn.com/2020/05/07/uk/uk-coronavirusethnicity-deaths-ons-scli-gbr-intl/index.html

22. Sama IE, Voors AA. Men more vulnerable to COVID-19: explained by ACE2 on the X chromosome? Eur Heart J. 2020;41(32):3096. doi:10.1093/ eurheartj/ehaa526

23. Tikellis $\mathrm{C}$, Thomas MC. Angiotensin-converting enzyme 2 (ACE2) is a key modulator of the renin angiotensin system in health and disease. Int J Pept. 2012;2012:256294. doi:10.1155/2012/256294

24. Molteni M. Meet ACE2, the enzyme at the center of the Covid-19 mystery. WIRED. June 1, 2020. Accessed September 29, 2020. https://www.wired com/story/meet-ace2-the-enzyme-at-the-center-of-the-covid-19-mystery/

25. Hashimoto T, Perlot T, Rehman A, et al. ACE2 links amino acid malnutrition to microbial ecology and intestinal inflammation. Nature. 2012;487(7408): 477-481. doi:10.1038/nature11228

26. Qian Z, Travanty EA, Oko L, et al. Innate immune response of human alveolar type II cells infected with severe acute respiratory syndrome-coronavirus. Am J Respir Cell Mol Biol. 2013;48(6):742-748. doi:10.1165/rcmb.2012O3390C

27. Bonafè M, Prattichizzo F, Giuliani A, Storci G, Sabbatinelli J, Olivieri F. Inflamm-aging: why older men are the most susceptible to SARS-CoV-2 complicated outcomes. Cytokine Growth Factor Rev. 2020;53:33-37. doi:10.1016/j.cytogfr.2020.04.005

28. Batlle D, Jose Soler M, Ye M. ACE2 and diabetes: ACE of ACEs? Diabetes. 2010;59(12):2994-2996. doi:10.2337/db10-1205

29. A Clinical Trial of Nebulized Surfactant for the Treatment of Moderate to Severe COVID-19 (COVSurf). ClinicalTrials.gov identifier: NCT04362059. Updated September 9, 2020. Accessed September 29, 2020. https://clinicaltrials.gov/ct2/show/NCT04362059

30. Bouie J. Why coronavirus is killing African-Americans more than others New York Times Sunday Review. April 19, 2020. Accessed September 29, 


\section{Angiotensin-Converting Enzyme 2, Feedback Systems, and COVID-19}

2020. https://www.nytimes.com/2020/04/14/opinion/sunday/coronavirusracism-african-americans.html

31. Bernstein KE, Khan Z, Giani JF, Cao D-Y, Bernstein EA, Shen XZ. Angiotensin-converting enzyme in innate and adaptive immunity. Nat Rev Nephrol. 2018;14(5):325-336. doi:10.1038/nrneph.2018.15

32. Risitano AM, Mastellos DC, Huber-Lang M, et al. Complement as a target in COVID-19? Nat Rev Immunol. 2020;20(6):343-344. doi:10.1038/s41577020-0320-7

33. Coronavirus 2019 (COVID-19)_Using Ascorbic Acid and Zinc Supplementation (COVIDAtoZ). ClinicalTrials.gov identifier: NCT04342728. Updated August 14, 2020. Accessed September 29, 2020. https://clinicaltrials.gov/ct2/ show/NCT04342728

34. Flack JM, Mensah GA, Ferrario CM. Using angiotensin converting enzyme inhibitors in African-American hypertensives: a new approach to treating hypertension and preventing target-organ damage. Curr Med Res Opin. 2000; 16(2):66-79.

35. Angiotensin-converting enzyme (ACE) inhibitors. Mayo Clinic. August 20, 2019. Accessed September 29, 2020. https://www.mayoclinic.org/diseasesconditions/high-blood-pressure/in-depth/ace-inhibitors/art-20047480

36. Kumar A, Kubota Y, Chernov M, Kasuya $\mathrm{H}$. Potential role of zinc supplementation in prophylaxis and treatment of COVID-19. Med Hypotheses. 2020; 144:109848. doi:10.1016/j.mehy.2020.109848

37. Zhong J-C, Huang D-Y, Yang Y-M, et al. Upregulation of angiotensin-converting enzyme 2 by all-trans retinoic acid in spontaneously hypertensive rats. Hypertension. 2004;44(6):907-912. doi:10.1161/01.HYP.0000146400. 57221.74

38. Wan Y, Shang J, Graham R, Baric RS, Li F. Receptor recognition by the novel coronavirus from Wuhan: an analysis based on decade-long structural studies of SARS coronavirus. J Virol. 2020;94(7):e00127-20. doi:10.1128/ JVI.00127-20

39. What you should know about ibuprofen and COVID-19. GSK Consumer Healthcare. Accessed September 29, 2020. https://www.gskhealthpartner. com/en-us/pain-relief/brands/advil/covid19/

40. Zhang P, Zhu L, Cai J, et al. Association of inpatient use of angiotensin-converting enzyme inhibitors and angiotensin II receptor blockers with mortality among patients with hypertension hospitalized with COVID-19. Circ Res. 2020;126(12):1671-1681. doi:10.1161/CIRCRESAHA.120.317134

41. Magrone T, Magrone M, Jirillo E. Focus on receptors for coronaviruses with special reference to angiotensin-converting enzyme 2 as a potential drug target-a perspective. Endocr Metab Immune Disord Drug Targets. 2020 20(6):807-811. doi:10.2174/1871530320666200427112902

42. Batlle D, Wysocki J, Satchell K. Soluble angiotensin-converting enzyme 2: potential approach for coronavirus infection therapy? Clin Sci (Lond). 2020; 134(5):543-545. doi:10.1042/CS20200163

43. Hanff TC, Harhay MO, Brown TS, Cohen JB, Mohareb AM. Is there an association between COVID-19 mortality and the renin-angiotensin system? A call for epidemiologic investigations. Clin Infect Dis. 2020;71(15):870-874. doi:10.1093/cid/ciaa329

44. Gralinski LE, Sheahan TP, Morrison TE, et al. Complement activation contributes to severe acute respiratory syndrome coronavirus pathogenesis. mBio. 2018.9(5):e01753-18 doi:10.1128/mBio.01753-18

45. Janeway CA Jr, Travers P, Walport M, Shlomchik M. The complement system and innate immunity In: Janeway CA Jr, Travers P, Walport M, Shlomchik M. Immunobiology: The Immune System in Health and Disease. 5th ed. Garland Science; 2001. Accessed September 29, 2020. https://www. ncbi.nlm.nih.gov/books/NBK27100/
46. Stoermer KA, Morrison TE. Complement and viral pathogenesis. Virology. 2011;411(2):362-373. doi:10.1016/j.virol.2010.12.045

47. Risitano AM, Mastellos DC, Huber-Lang M, et al. Complement as a target in COVID-19? Nat Rev Immunol. 2020;20(6):343-344. doi:10.1038/s41577 020-0320-7

48. Oster G, Salgo MP. Copper in mammalian reproduction. Adv Pharmacol Chemother. 1977;14:327-409. doi:10.1016/s1054-3589(08)60191-X

49. Zinc: fact sheet for consumers. National Institutes of Health Office of Dietary Supplements. Updated July 15, 2020. Accessed September 29, 2020. https://ods.od.nih.gov/factsheets/Zinc-Health\%20Professional/

50. Pan P-P, Zhan Q-T, Le F, Zheng Y-M, Jin F. Angiotensin-converting enzymes play a dominant role in fertility. Int J Mol Sci. 2013;14(10):21071-21086. doi:10.3390/ijms141021071

51. Hagaman JR, Moyer JS, Bachman ES, et al. Angiotensin-converting enzyme and male fertility. Proc Natl Acad Sci U S A. 1998;95(5):2552-2557. doi:10.1073/pnas.95.5.2552

52. Rivière G. Angiotensin-converting enzyme: a protein conserved during evolution [in French]. J Soc Biol. 2009;203(4):281-293. doi:10.1051/jbio/ 2009032

53. Ocampo Daza D, Larhammar D. Evolution of the growth hormone, prolactin, prolactin 2 and somatolactin family. Gen Comp Endocrinol. 2018;264:94 112. doi:10.1016/j.ygcen.2018.01.007

54. Shu Y, Lou Q, Dai Z, et al. The basal function of teleost prolactin as a key regulator on ion uptake identified with zebrafish knockout models. Sci Rep. 2016;6:18597. doi:10.1038/srep18597

55. Brook CE, Boots M, Chandran K, et al. Accelerated viral dynamics in bat cell lines, with implications for zoonotic emergence. Elife. 2020;9:e48401. doi:10.7554/eLife.48401

56. Jabr F. How humanity unleashed a flood of new diseases. New York Times Magazine. June 17, 2020. Updated June 25, 2020. Accessed September 29, 2020. https://www.nytimes.com/2020/06/17/magazine/animal-diseasecovid.html

57. Maxwell JC. On governors. Proc Royal Soc Lond. 1868;16:270-283. Accessed September 29, 2020. https://www.jstor.org/stable/pdf/112510.pdf

58. Hellerstein JL, Diao Y, Parekh S, Tilbury DM. Feedback Control of Computing Systems. Wiley-IEEE Press; 2004.

59. Basic introduction to feedback control. Electrical Engineering and Compute Science, University of Michigan. Accessed September 29, 2020. https:// www.eecs.umich.edu/courses/eecs373.w05/lecture/control.htm

60. Baraniuk R, Kruzick S. BIBO stability of continuous time systems. Signals and systems. Connexions 2.0. Accessed September 29, 2020. http://pilot. cnxproject.org/content/collection/col10064/latest

61. Guillaud F, Hannaert P. A computational model of the circulating renin-angiotensin system and blood pressure regulation. Acta Biotheor. 2010;58(23):143-170. doi:10.1007/s10441-010-9098-5

62. Barbiero P, Lió P. The computational patient has diabetes and a COVID. Preprint. Posted online July 20, 2020. medRxiv. doi:10.1101/2020.06.10. 20127183

63. Liu J, Chen T, Yang H, et al. Clinical and radiological changes of hospitalised patients with COVID-19 pneumonia from disease onset to acute exacerbation: a multicentre paired cohort study. Eur Radiol. 2020;1-7. doi:10.1007/ s00330-020-06916-4

64. OER Services. Anatomy and Physiology I, Module 2. Homeostasis and feedback loops. Accessed October 5, 2020. https://courses.lumenlearning.com/ suny-ap1/chapter/homeostasis-and-feedback-loops/ 